\title{
Measuring pressure during coronary artery angiography in ex-vivo hearts
}

\begin{abstract}
Coronary artery disease (CAD) is the most common cause of sudden adult death. Diagnosis in life and after death is therefore crucial, but can be problematic and inaccurate. Postmortem CT angiography (PMCTA) is attempting to address this, but its accuracy is still not fully established. In clinical practice, pressure measurements, recording drops in pressure across the stenosis, are now being used to determine physiological significance, as the degree of narrowing on imaging can be misleading. This study was designed to investigate the introduction of pressure measurement to PMCTA in order to understand the importance of re-pressurising vessels in the evaluation of CAD.
\end{abstract}

Ex-vivo porcine hearts were used to develop the technique. Methods to introduce catheters and wires were investigated and a system was developed to suspend the heart, to enable pressure tests and CT scans.

Consistent measurable pressures were achieved with good correlation of measured arterial pressure to delivered pressure in most cases. Pressure measurements were shown to be inaccurate in decomposed hearts, poor dissection and cannulation damaging the vessel, contamination of the vessels (e.g. by air) and malposition of the pressure measurement wire.

\section{INTRODUCTION}

Coronary artery disease (CAD) is the most common cause of adult sudden death and its evaluation in post mortem practice is therefore essential. The introduction of angiography to the post-mortem evaluation of CAD has allowed coronary artery disease to be assessed accurately in the deceased [1, 2]. This was a significant advance and has increased the possibility of using PMCT to replace autopsy for adult natural death [3, 4]. Imaging is important to the clinical evaluation of patients with CAD and traditional angiography has been the 'gold standard' for decades [5]. Cardiac CT angiography (CCTA) is now available to most cardiology units with the introduction of spiral CT with $16+$ detectors.

However, all contrast angiography methods are limited by two key problems: firstly, their inability to image the vessel wall and secondly, the degree of stenosis may not always directly correlate with its functional significance [6]. Clinically angiography can over- and under-estimate the extent of narrowing and distort vessels by foreshortening or elongating images in up to $70 \%$ of patients $[5,7]$. Studies also show up to $50 \%$ inter and intra operator variability in the assessment of stenosis on angiographic images $[5,8]$. 
Post mortem computed tomography angiography (PMCTA) has been developed based on clinical advances. The improvements PMCTA has made to the diagnosis of CAD have been widely discussed $[9,10,11,12]$ and its accuracy verified against histological studies [13]. Using pressurised injections in PMCTA in order to replicate physiological conditions has been described $[11,12]$. Pressures have been measured in PMCTA studies, with the intention to limit pressure to less than $60 \mathrm{mmHg}$ to avoid vascular extravasation [11] and iatrogenic vascular rupture [14]. Others have advocated the use of higher pressures to simulate vital blood pressure [15].

Fractional flow reserve (FFR) is a measurement based on blood pressure differences caused by stenoses. The pressures are measured using a very small transducer delivered on a thin vascular wire. The FFR has been shown to be a more reliable indicator of the success of revascularisation than an observed narrowing alone [6], changing the focus of coronary artery intervention onto physiologically significant stenoses, thereby reducing risk to the patient and cost to health care providers [16]. In the deceased, FFR cannot be measured physiologically as there is no natural blood pressure. However, by re-pressurising the cadaveric vessels, a correlate of FFR can theoretically be created.

This study was devised to create a system for ex-vivo re-pressurisation of coronary arteries and directly measure the pressures obtained within the artery. This would allow measurement of pressure differences across stenoses, and therefore estimation of the FFR and therefore the physiological significance of a stenosis.

\section{MATERIALS AND METHODS}

Ethical approval has been granted for this work (LREC 04/Q2501/64, UHL 09523) and the larger on-going project.

Porcine model: Testing was undertaken using porcine hearts obtained from a local abattoir. Initially, in accordance with UK law at the time, the hearts were examined for parasites, which involved slicing into the tissues. Some vessels were damaged in this process, but only 1 heart had neither coronary artery intact. The hearts were washed before being packaged in plastic containers for transportation. Hearts not used on the day of collection were refrigerated or frozen depending on the interval before use.

Technique: The left main stem (LMS) and right coronary artery (RCA) ostia were located and a $6 \mathrm{Fr}$ coronary catheter was inserted. Fat around the exterior of the vessel was removed to enable a stitch to secure the cannula. A 0.035 inch guide wire was inserted down the $6 \mathrm{Fr}$ catheter as distally as possible and a microcatheter (Finecross ${ }^{\circledR}$ MG, Terumo) passed over it. The guide wire was then replaced with a pressure wire and the microcatheter was removed. The pressure wire (PressureWire ${ }^{\mathrm{TM}}$ Certus $^{\mathrm{TM}}$, St. Jude Medical, Inc.) has a pressure measurement transducer $3 \mathrm{~cm}$ from its flexible tip. The flexible tip of the pressure wire coiled in some vessels and so it was cut to reduce the tip to $0.5 \mathrm{~cm}$ long. An Optical Coherence Tomography (OCT) catheter (Dragonfly Duo ${ }^{\mathrm{TM}}$ St Jude Medical) was then passed through the 6Fr catheter, over the pressure wire into the vessel ensuring the transducer on the pressure wire was not covered, to allow the luminal diameter to be measured. 
Initially pressure wires were inserted directly into vessels, but OCT imaging showed intimal dissection could be caused easily without using the microcatheter (figure 1).

A " $Y$ " connector (Speedketch, Minvasys) enabled a sealed system to be used, with the wires passing through one port and pressurised fluid infused through the other. Pressure was generated using a sphygmomanometer around a saline bag ${ }_{\bar{j}}$ and "delivered" pressure was measured by a transducer connected between the fluid giving set and the " $Y$ " connector. The transducer and pressure wire were connected to a pressure recording system (Radianalyzer, St Jude Medical Systems). This approach has similarities to one previously described [17]. Three pressures could be recorded, the pressure generated by the sphygmomanometer ("cuff" pressure), the delivered pressure, recorded the level of the " $Y$ " connector (often called $\mathrm{Pa}$, the aortic pressure in clinical systems) and the pressure in the coronary artery (Pd). Pd could be measured in the proximal, mid or distal coronary artery. $\mathrm{Pa}$ and $\mathrm{Pd}$ were the studied pressures as the generated pressure, as measured by the sphygmomanometer is not accurate.

Time and pressure of infusion: The pressures generated and the time to reach maximum pressure at different infusion pressures was studied. $\mathrm{Pa}$ and $\mathrm{Pd}$ pressures were recorded at the start of the infusion and at time intervals of up to 5 minutes, with cuff pressures ranging from 10 to $150 \mathrm{mmHg}$. This was later simplified to recording pressures at $0,15,30$ and 60 seconds with the infusion stopping at 30 seconds. Fluid was infused at $10 \mathrm{mmHg}$ increments from 10 to $100 \mathrm{mmHg}$ and 120 and $150 \mathrm{mmHg}$. Each pressure test was repeated three times.

Apparatus: The effect of external pressure on vessels was minimised by suspending the heart in a container, with rod through a hole made in the pulmonary trunk. This rig also enables the hearts to be surrounded by water during scanning to minimise air-tissue interface artefacts. The rig has similarities to that previously described [18].

Imaging: OCT images were obtained using a Dragonfly Duo ${ }^{\mathrm{TM}}$ OCT catheter connected to an llumien $^{\mathrm{TM}} \mathrm{PCl}$ Optimization system (both from St Jude Medical) and the images were taken as the OCT catheter was withdrawn.

One heart was CT scanned to look at vessel filling and contrast in the myocardium. Both left and right coronary arteries were cannulated as above. A Toshiba Aquilion One scanner was used. The heart was placed in the rig as for the pressure testing. The container was filled with water and the lid fitted to minimise the risk of water leaking on to the scanner. The catheters were routed out of holes in the sides of the container. Scans used $100 \mathrm{kVp}, 80 \mathrm{~mA}$, 0.35 rotation time. An initial scan was performed to check the position of the catheters. $15 \%$ Urografin ${ }^{\circledR}$ (Bayer Healthcare) diluted with water was then injected using a Medrad Stellant pump injector system (Medrad UK Ltd) at $0.5 \mathrm{mls} / \mathrm{sec}$. A slow infusion rate was used to minimise risk of catheter displacement. Each vessel was scanned every 1.8 seconds for 61.2 seconds following a 5 second scan delay. On completion of the scan the injector was stopped and connected to the catheter in the other vessel and the process repeated. $36 \mathrm{mls}$ of contrast was used for each injection. 
Statistics: Where means are compared $95 \%$ confidence intervals are calculated. " $\mathrm{P}$ " values are derived using Students T test. Within-subject standard deviation, measurement error and repeatability are calculated using the method described by Bland \& Altman [19].

\section{RESULTS}

Fifteen pig hearts were used from pigs less than 12 months old. 1 heart had both pressure studies and CT scan. No coronary artery disease was seen in any of the pig hearts used on inspection and OCT imaging.

Of the 15 pig hearts for pressure studies 1 was excluded as both coronary arteries had been cut during the abattoir parasite check, 1 was excluded due to decomposition from post mortem interval and re-freezing and 3 were excluded due to technical failure to cannulate either vessels or damaging the vessel in doing so. This left 10 pig hearts in the study.

Initial testing demonstrated maximal pressure wire reading (within $3 \mathrm{mmHg}$ in all cases) was achieved by 30 seconds. Therefore the rest of the results are measured at 30 seconds.

Pressures were recorded in both the LAD and RCA of two hearts, in the LAD only in 9 hearts and RCA only in 3 hearts. In the LAD vessels 12 runs were performed, 4 with the pressure wire in the proximal vessel, 1 in the mid vessel and 7 in the distal vessel. In the RCA vessels 5 runs were performed, 2 with the pressure wire in the proximal vessel, 2 in the mid vessel and 1 in the distal vessel. In two cases the run was repeated in the same vessel position, one after refilling the vessel with fluid and one after repositioning the catheter

Four runs showed obvious discrepancies between delivered pressure and measured artery pressure. In one case the pressure recorded in the coronary artery was higher than delivered pressure, but corrected suddenly to better agreement as the delivered pressure increased (figure 2). This was judged at the time to be due to an air bubble adjacent to the transponder that moved with the increasing pressure. In 3 cases the measured coronary artery pressure was significantly below delivered pressure. In 2 cases this difference was greater for distal artery measurements compared with in the proximal artery (figure 3 ). In the other re-positioning the measurement probe resulted in better agreement of delivered and measured pressure (figure 4).

In the other 13 runs good correlation was achieved between delivered and measured coronary artery pressure (figure 5). The mean delivered pressure measured by the proximal transponder was $57.9 \mathrm{mmHg}(\mathrm{SD}=29.0 \mathrm{mmHg}$ ) and the mean pressure measured in the coronary artery was $51.4 \mathrm{mmHg}$ (SD $=28.6 \mathrm{mmHg}$ ). Despite good correlation these data do show a systematic reduction in measured artery pressure compared with delivered pressure with mean measured pressure $6.5 \mathrm{mmHg}$ less than delivered pressure $(95 \% \mathrm{Cl}=0.58 \mathrm{mmHg}$, $p<0.0001)$. The degree of difference between measured and delivered pressure is not related to magnitude of pressure. No specific reason for this was seen but in clinical practice the blood pressure measured by both the proximal transponder and pressure wire would be equalised in the aorta, which may explain some of the difference. 
If this systematic underestimation is taken into account the within-subject standard deviation is $4.1 \mathrm{mmHg}$ giving a measurement error of $8.1 \mathrm{mmHg}$ (95\% of repeated measurements will be within $8.1 \mathrm{mmHg}$ of the true value), with a 95\% repeatability of 11.4 $\mathrm{mmHg}$ (95\% of repeated measurements will be within $11.4 \mathrm{mmHg}$ of each other).

\section{DISCUSSION}

These data show that it is possible to re-pressurise the coronary arteries ex-vivo and measure coronary artery pressures. Care has to be taken at cannulation, as measurements may be inaccurate if the vessel is damaged, there is air within the vessel or the pressure wire is sited incorrectly.

Therefore dissection of the vessel and cannulation has to be performed with care with a microcatheter rather than direct access with the pressure wire. In 2 cases the pressure wire became wedged in the vessel and failed to measure the pressure. In clinical practice this is a rare, but recognised complication of angiographic procedures, but the lack of blood flow in the ex-vivo hearts exacerbated this problem. The pressure wires have a $3 \mathrm{~cm}$ long flexible tip that in clinical patients would be carried into the coronary ostia by the blood flow. Without blood flow the long flexible tip caused cannulation difficulties, and so it was shortened. This increased the risk of the wire perforating the vessel but if the wire was positioned using the microcatheter this did not occur.

Air can already be in the arteries or can be introduced when the wires and catheters were inserted. If seen, the vessel was flushed with water either through a micro-catheter passed over the pressure wire or the OCT catheter. The displaced air bubbles were observed leaving the $Y$ connector. Flushing the vessel through the OCT catheter, prior to starting any testing, became routine and helped reduce pressure irregularity.

Equipment is reused whenever possible and pressure wires are reused as often as possible due to their high cost, but vigilance for unusual pressures was needed to indicate a failing wire.

Many of the porcine hearts were incised for the parasite check and so vessels were damaged. A damaged heart, even to the microvasculature, would not be expected to maintain arterial pressure in the same was as an intact system. This may not always be obvious on initial inspection but may be seen using contrast enhanced CT scanning (figure $6)$.

There is a systematic underestimation of pressures in the coronary artery compared with the delivered pressure. It is possible that this could be corrected by 'equalising' between the two transponders before the measurement runs. This cannot be done in the same way as for clinical studies (in the aorta), but could be done with the catheter in the proximal coronary artery before passage into the distal vessel. The pressure drop could also be related to the use of an ex-vivo porcine system. However, the main purpose of the measurement would be to detect pressure drops across a stenosis within the vessel, which 
is unrelated to the actual delivered pressure, as long as this remains constant. Therefore, this should not affect the measurement of any pressure drop across a stenosis.

Measurement error is calculated as $+/-8.1 \mathrm{mmHg}$ and $95 \%$ repeatability as $11.1 \mathrm{mmHg}$. In clinical practice a blood pressure drop of greater than $20 \%$ (FFR $=0.8$ ) would be considered significant. This would correspond to about $25 \mathrm{mmHg}$ at normal blood pressure (or greater in hypertension). The mean delivered pressure was $57.9 \mathrm{mmHg}$ and mean coronary artery pressure was $51.4 \mathrm{mmHg}$, but pressures up to $120 \mathrm{mmHg}$ were achievable. Therefore, theoretically this technique would be capable of measuring clinically significant pressure differences, if this pressure can be achieved in-vivo.

The use of porcine hearts was helpful to develop the techniques. However these are not directly comparable to human hearts and did not have coronary artery disease so this study does not assess the impact of an atheromatous stenosis on pressure measurement. Comparison with clinical pressure studies is also problematic as the flow is continuous and not pulsatile and the effect of myocardial contractility cannot be replicated. These issues will constitute the next phase of this work on ex-vivo human hearts and ultimately in-vivo cadaveric hearts. A pilot study has shown that it is possible to cannulate the coronary arteries and perform angiography and OCT in cadavers but the techniques need to be perfected [20].

\section{CONCLUSION}

In summary we have developed a technique that can re-pressurise and measure the pressure generated in ex-vivo coronary arteries in a porcine model that we are now translating to explanted human hearts. We hope to be able to further translate this technique into intact hearts in the cadaver. By measuring pressure gradients we can study the potential significance of observed vessel stenoses and also develop techniques of predicting pressure gradients.

\section{FIGURES}

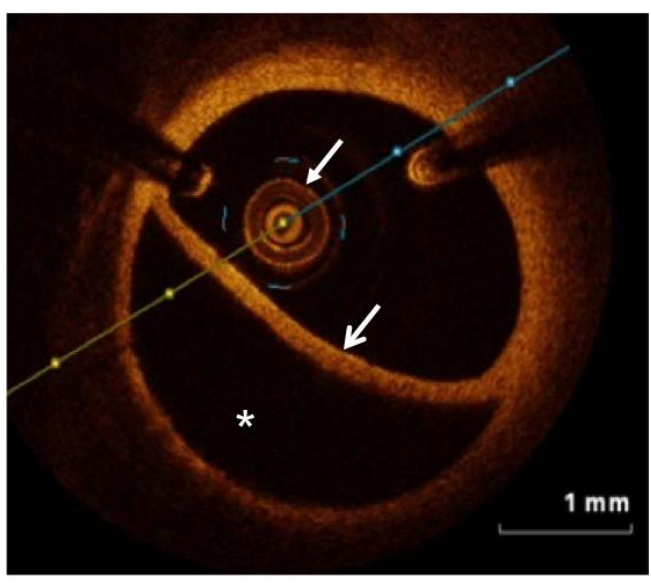


Figure 1. Optical Coherence Tomography (OCT) image showing a dissected vessel. Closed arrow indicates OCT probe in the true lumen. The dissection flap (open arrow) separates the true lumen from the false lumen (asterisk).

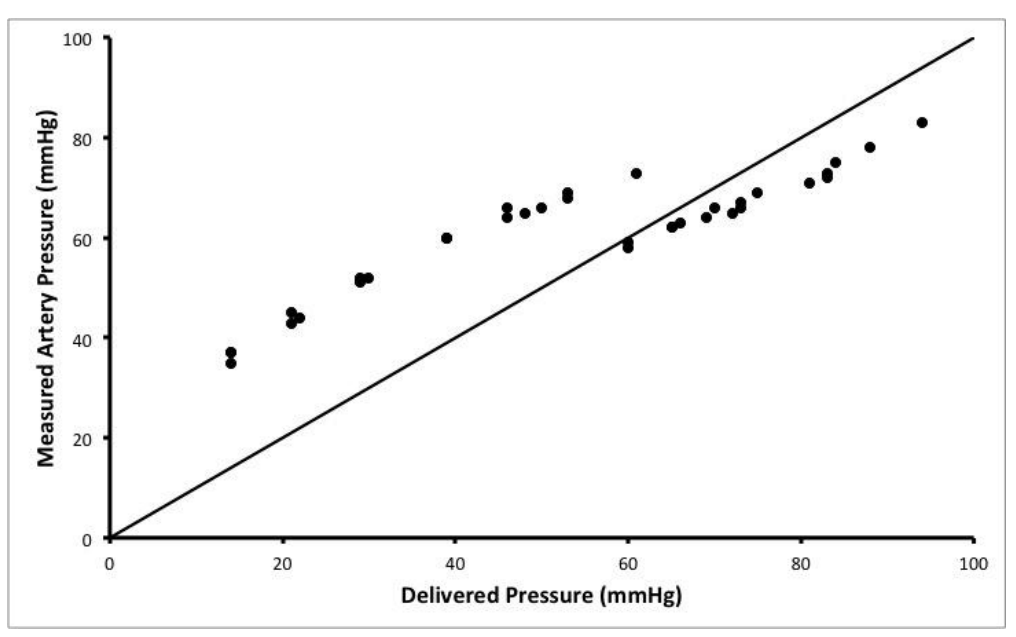

Figure 2. Plot of artery pressure, measured by the pressure wire transducer, against delivered pressure, measured by the transponder in the giving-set, for a single series. Black line shows identity. The measured pressure was higher than expected initially and then corrected after one of the measurements at $60 \mathrm{mmHg}$. This was believed to be due to an air bubble adjacent to the transponder on the pressure wire, which cleared during the experiment.

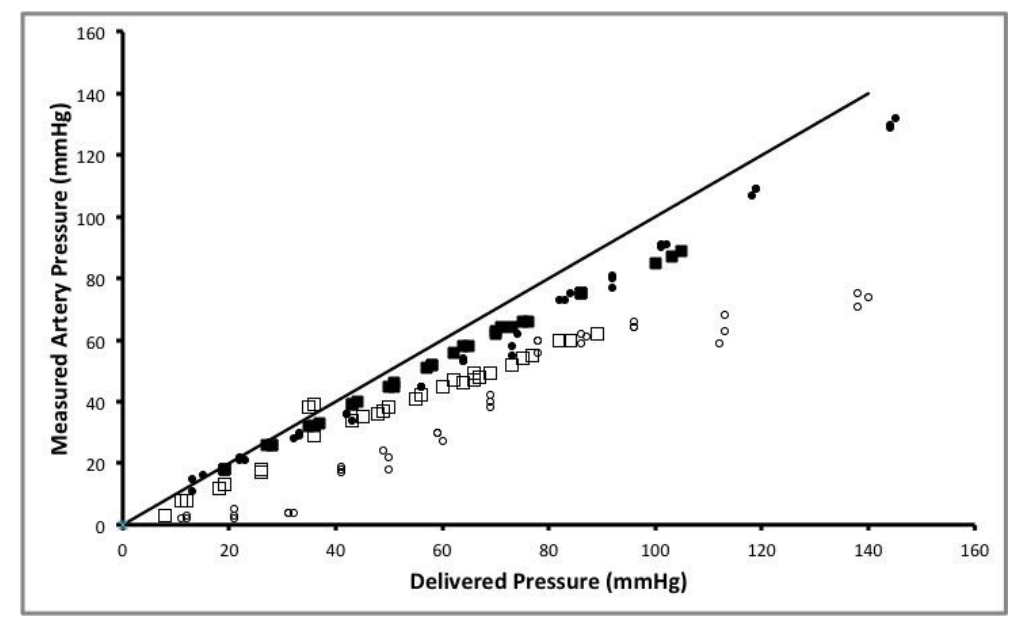

Figure 3. Plot of artery pressure, measured by the pressure wire transducer, proximally (filled shapes) and distally (unfilled shapes), against the delivered pressure, measured by the transponder in the giving-set, for two different hearts (squares and circles). Black line shows identity. This shows good correlation of measured to delivered pressures proximally, but a pressure drop occurring distally. This was assumed to be due to air or other debris in the vessel. 


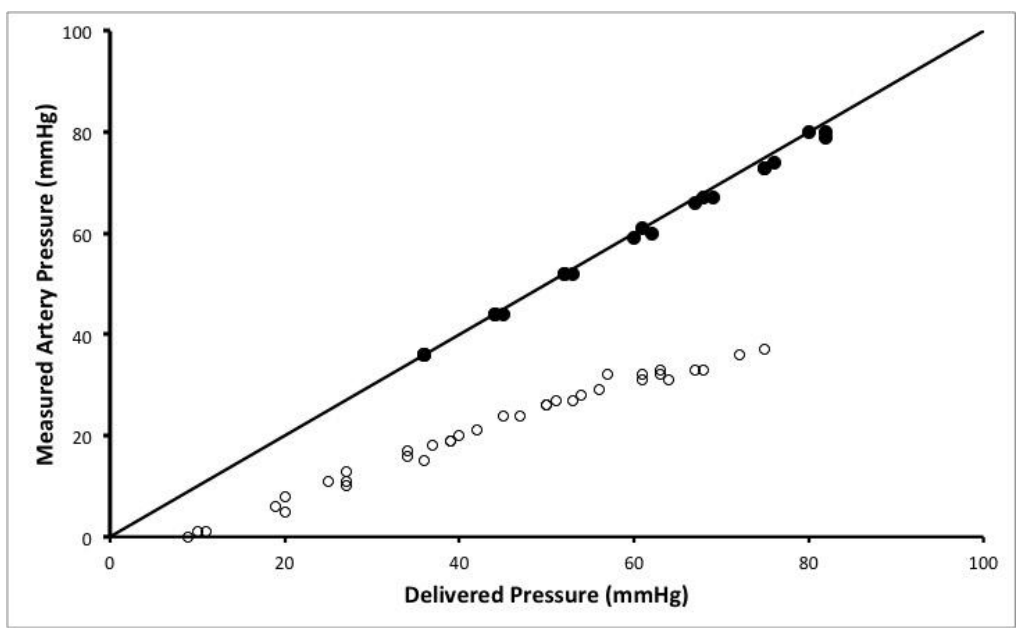

Figure 4. Plot of artery pressure, measured by the pressure wire transducer, against delivered pressure, measured by the transponder in the giving-set, for two runs on the same heart. The first run (clear circles) gave poor correlation of measured to delivered pressure. This was considerably improved by repositioning the pressure wire (solid circles).

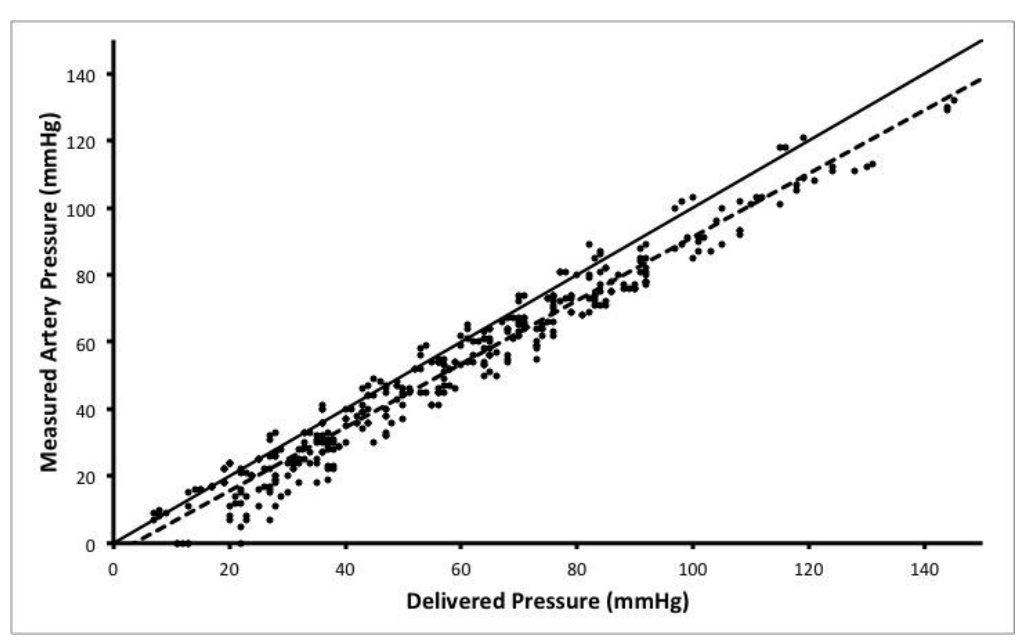

Figure 5. Plot of artery pressure, measured by the pressure wire transducer, against delivered pressure, measured by the transponder in the giving-set, for the 13 runs without overt measurement problem. Solid Black line shows identity and dashed line is the data fit line $\left(R^{2}=0.96\right)$. 


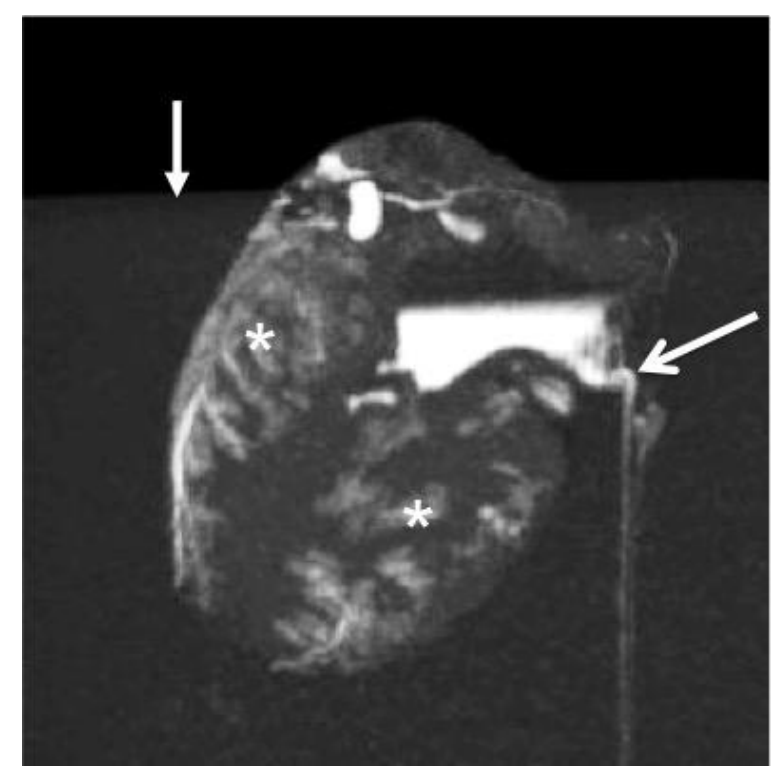

Figure 6. CT scan Maximum Intensity Projection (MIP) of an ex vivo pig heart after contrast injection. Closed arrow shows the water level in the container. There is a contrast leak (open arrow) that may have contributed to some loss of pressure. There is also contrast enhancement of the myocardium (white stars) consistent with extra vascular leakage of contrast, as is seen in clinical CT scanning.

\section{REFERENCES}

1. Saunders SL, Morgan B, Raj V, Robinson C, Rutty GN. Targeted post-mortem computed tomography cardiac angiography: proof of concept. International Journal of Legal Medicine 2011, 125(4):609-616. DOI 10.1007/s00414-011-0559-4.

2. Grabherr S, Gygax E, Sollberger B, Ross S, Oesterhelweg L, Bolliger S, Christe A, Djonov V, Thali M J, Dirnhofer R. Two-Step Postmortem Angiography with a Modified Heart-Lung Machine: Preliminary Results. American Journal of Roentgenology, 2008, 190 (2) 345-351. DOI 10.2214/AJR.07.2261.

3. Morgan B, Biggs M J, Barber J, Raj V, Amoroso J, Hollingbury F E, Robinson C, Rutty G N. Accuracy of targeted post-mortem computed tomography coronary angiography compared to assessment of serial histological sections International Journal of Legal Medicine, 2013, 127:809-817. DOI 10.1007/s00414-012-0790-7.

4. Roberts IS D, Trail Z C. Minimally invasive autopsy employing post-mortem CT and targeted coronary angiography: evaluation of its application to a routine Coronial service. Histopathology, 2014, 64, 211-217. DOI: 10.1111/his.12271.

5. Topol E J, Nissen S E. Our preoccupation with coronary luminology. Circulation, 1995, 92, 2333-2342. DOI 10.1161/01.CIR.92.8.333.

6. De Bruyne B, Sarma J. Fractional flow reserve: a review. Heart, 2008, 94, 949-959. DOI 10.1136/hrt.2007.122838 
7. Isner J M, Kishel J, Kent K M, Ronan J A, Ross A M, Roberts W C. Accuracy of angiographic determination of left main coronary arterial narrowing. Circulation, 1981, 63 (5) 1056-1064. DOI 10.1161/01.CIR.63.5.1056.

8. Gould K L. Quantification of coronary artery stenosis in vivo. Circulation Research, 1985, 57 (3) 341-353. DOI 10.1161/01.RES.57.3.341.

9. Michaud K, Grabherr S, Doenz F, Mangin P. Evaluation of postmortem MDCT and MDCTangiography for the investigation of sudden cardiac death related to atherosclerotic coronary artery disease. International Journal of Cardiovascular Imaging, 2012, 28(7), 18071822. DOI 10.1007/ s10554-012-0012-x.

10. Roberts I S D, Benamore R E, Peebles C, Roobottom C, Trail Z C. Diagnosis of coronary artery disease using minimally invasive autopsy: evaluation of a novel method of post-mortem coronary CT angiography. Clinical Radiology 2011, 66 (7) 64650. DOI 10.1016/j.crad.2011.01.007.

11. Jackowski C, Sonnenschein M, Thali M J, Aghayev E, von Allmen G, Yen K, Dirnhofer R, Vock P. Virtopsy: postmortem minimally invasive angiography using cross section techniquesimplementation and preliminary results. Journal of Forensic Science, 2005, 50 (5) 11751186. DOI: $10.1520 / J F S 2005023$.

12. Morgan B, Sakamoto N, Shiotani S, Grabherr S. Postmortem Computed Tomography (PMCT) Scanning with Angiography (PMCTA): A Description of Three Distinct Methods in Rutty G N (ed) Essentials of Autopsy Practice, Springer, London, 2014, pp 1-21. DOI 10.1007/978-14471-5270-5_1.

13. Morgan B, Biggs M J, Barber J, Raj V, Amoroso J, Hollingbury F E, Robinson C, Rutty G N. Accuracy of targeted post-mortem computed tomography coronary angiography compared to assessment of serial histological sections. International Journal of Legal Medicine, 2013, 127 (4) 809-817. DOI 10.1007/s00414-012-0790-7.

14. Berger N, Martinez R, Winklhofer S, Flach P M, Ross S, Ampanozi G, Gascho D, Thali M J, Ruder T D. Pitfalls in post-mortem CT-angiography - intravascular contrast induces postmortem pericardial effusion. Legal Medicine. 2013, 15, 315-317. DOI 10.1016/j.legalmed.2013.07.001.

15. Pøhlsgaard C and Leth P M. Post-mortem CT-coronary angiography. Scandinavian Journal of Forensic Sciences 2007, 1, 1-32.

16. Pijls N H J, Fearon W F, Tonino P A, Siebert U, Ikeno F, Bornschein B, van't Veer M, Klauss V, Manoharan G, Engstrøm T. Fractional flow reserve versus angiography for guiding percutaneous coronary intervention in patients with multivessel coronary artery disease. Journal of the American College of Cardiology, 2010, 56 (3) 177-184. DOI 10.1016/j.jacc.2010.04.012.

17. Inokuchi G, Yajima D, Hayakawa M, Motomura A Chiba F, Torimitsu S, Makino Y, Iwase H. The utility of postmortem computed tomography selective coronary angiography in parallel with autopsy. Forensic Science Medicine and Pathology 2013, 9, 506-514. DOI 10.1007/s12024-013-9473-z. 
18. Johnson T W, White S, Gnanadesigan M, Bourenane H, Strange J W, Newby A C, van Soest G, Baumbach A. An ex-vivo 'whole human heart model' for the development of intravascular imaging. Heart 2012, 98 (supp 1) A15-A16. DOI:10.1136/heartjnl-2012-301877b.23

19. Bland JM, Altman DG. Statistical Methods For Assessing Agreement Between Two Methods Of Clinical Measurement. The Lancet 1986, 327, (8476), 307-310. doi:10.1016/S01406736(86)90837-8.

20. Adlam D, Joseph S, Robinson C, Rousseau C, Barber J, Biggs M, Morgan B, Rutty G. Coronary optical coherence tomography: minimally invasive virtual histology as part of targeted postmortem computed tomography angiography. International Journal of Legal Medicine 2013, 127, 991-6. DOI 10.1007/s00414-013-0837-4. 